\title{
Does aquatic exercise improve commonly reported predisposing risk factors to falls within the elderly? A systematic review
}

\author{
Eduardo Martínez-Carbonell Guillamón ${ }^{1,2^{*}} \mathbb{D}$, Louise Burgess², Tikki Immins², \\ Andrés Martínez-Almagro Andreo ${ }^{1}$ and Thomas W. Wainwright ${ }^{2}$
}

\begin{abstract}
Background: According to the World Health Organization, the elderly are at the highest risk of injury or death from a fall. Age-related changes in strength, balance and flexibility are degenerative factors that may increase the risk of falling, and an aquatic training may offer a favourable environment to improve these modifiable risk factors.

Methods: A systematic review was conducted to assess the potential preventative role of aquatic exercise for reducing the risk of falls in the elderly by improving predisposing risk factors. Electronic databases and reference lists of pertinent articles published between 2005 and 2018 were searched. Randomized controlled trials (RCTs) that directly or indirectly addressed the effect of aquatic exercise for the prevention of falls in healthy participants were included within the synthesis. Studies were included if they were reported between January 2005 and May 2018 within a population aged between 60 and 90 years old that were without exercise-effecting comorbidities. Data related to participant demographics, study design, methodology, interventions and outcomes was extracted by one reviewer. Methodological quality assessment was independently performed by two reviewers using the PEDro (Physiotherapy Evidence Database) scale.

Results: Fourteen trials met the inclusion criteria. Exercise intervention duration and frequency varied from 2 to 24 weeks, from 2 to 3 times per week, from 40 to 90 min per session. Fall rate was not reported in any of the studies analysed. However, aquatic exercise improved key predisposing physical fitness components that are modifiable and internal risk factors for falling.

Conclusions: There is limited, low-quality evidence to support the use of aquatic exercise for improving physiological components that are risk factors for falling. Although the evidence is limited, and many interventions are not well described, these results should be considered by health and exercise professionals when making evidence-based, clinical decisions regarding training programmes to reduce the risk of falling.
\end{abstract}

Keywords: Elderly, Aquatic exercise, Magnitude of load, Fall prevention

\section{Background}

Fall-related injuries and deaths are serious and increasing issues for the elderly population. A fall is defined as "an event which results in a person coming to rest inadvertently on the ground or floor or other lower level, excluding intentional change in position to rest in

\footnotetext{
* Correspondence: emartinez913@alu.ucam.edu

${ }^{1}$ Faculty of Health Science, Catholic University of Murcia, UCAM, Av. de los Jerónimos, 135, Guadalupe, 30107 Murcia, Spain

${ }^{2}$ Orthopaedic Research Institute, Bournemouth University, 6th Floor,

Executive Business Centre, 89 Holdenhurst Road, Bournemouth BH8 8EB, UK
}

furniture, wall or other objects" [1]. Approximately 30\% of people aged over 65 years will fall at least once a year $[2,3]$ and $15 \%$ at least twice a year [4], and this risk increases with age. These falls may result in fracture, long-term pain, disability and functional impairments and as a consequence, an individual may suffer negative social effects, due to a lack of independence, which reduces an individual's quality of life. In addition, falls in the elderly create an economic burden to hospitals and healthcare systems [5].

(c) The Author(s). 2019 Open Access This article is distributed under the terms of the Creative Commons Attribution 4.0 International License (http://creativecommons.org/licenses/by/4.0/), which permits unrestricted use, distribution, and reproduction in any medium, provided you give appropriate credit to the original author(s) and the source, provide a link to the Creative Commons license, and indicate if changes were made. The Creative Commons Public Domain Dedication waiver (http://creativecommons.org/publicdomain/zero/1.0/) applies to the data made available in this article, unless otherwise stated. 
There are a multitude of risk factors for falling, and they can be internal or external and modifiable or unmodifiable. Some risk factors are directly correlated and others interact in a more complex manner [2]. Ageing is associated with anatomical and physiological changes that can lead to increased disability, frailty and a higher risk of falls [6]. Sarcopenia is the degenerative loss of muscle mass, which increases linearly with age [7]. In addition, involuntary, age-related impairments of the three sensory systems that control posture (vestibular, visual and somatosensory) can lead to falls. A study by Rubenstein [8] reviewed 16 controlled studies and found weakness, balance deficit and gait deficit to be the most important individual risk factors for falls. More specifically, a systematic review [9] found lower extremity weakness to be a clinically important and statistically significant risk factor for falls. In addition, a study by Myers et al. [10] proposed strength, flexibility, balance and reaction time to be the most modifiable internal risk factors to falls, therefore providing a rationale for exercise interventions that aim to reduce falls in the elderly.

Research shows that detection and improvement of predisposing modifiable risk factors can reduce the rate of future falls [11, 12]. Regular physical exercise has been theorised to counteract the negative physiological effects of the aging process and improve physical and mental wellbeing [12, 13]. However, barriers to exercise behaviour among older adults include a fear of falling and perceived negative affect [14] and it is therefore difficult to conclude which training prescription (for example: load control training, progression of the load during balance exercises, type of equipment, depth of water) will create an effective programme $[15,16]$. Studies that have compared aquatic and land group with the same protocol, through specific functionality tests, do not report significant differences between training groups [17]. Therefore, it is possible that aquatic exercise may improve balance, strength and flexibility out of water despite the differences in the environment.

Aquatic exercise provides a low-impact and low-weight bearing environment where individuals can exercise safely. The risk of falling is eliminated, and therefore an individual can concentrate on making physical improvements. In addition to safety benefits, aquatic programs may offer an appealing alternative to repetitive, conventional exercise which may increase compliance to rehabilitation plans. There are many components of physical fitness that can be trained in an aquatic environment to help reduce the risk of falling, for example: agility, balance, co-ordination, strength, flexibility and speed. The aim of this systematic review is to evaluate the effectiveness of using an aquatic environment to reduce the most modifiable predisposing risk factors of falls within an elderly population. The type of exercise, frequency, intensity and duration of each exercise intervention will be assessed to determine if physical activity within an aquatic environment offers potential benefits to the risk of falling.

\section{Methods \\ Protocols}

This manuscript is written in accordance with the PRISMA (Preferred Reporting Items for Systematic Reviews and Meta-Analyses) statement which includes controlled and randomized studies [18, 19]. A systematic review was completed to assess the role of aquatic exercise for improving the commonly reported predisposing risk factors of falls in the elderly.

\section{Eligibility criteria and information sources}

A computer based search was completed between January 2005 and May 2018 and the electronic databases sourced were: ISI Web of Knowledge, ProQuest, PubMed, Science Direct, SPORTDiscus, the Cochrane Central Register of Controlled Trials and the Google Academic Meta searcher. The PICO (Population, Intervention, Comparison and Outcome) framework was used to define the search strategy. The search strategy was developed to include a combination of controlled vocabulary $(\mathrm{MeSH})$ and free text terms which can be found in Table 1. Reviews and commentaries were used to identify papers, but were not included within the synthesis of results. The search reviewed all available studies published between 2005 and 2018. In the mid-nineties, strong research emerged on the benefit of the aquatic environment in rehabilitation compared to the land environment. In particular, an article belong to Tovin [20] who compared the anterior cruciate ligament reconstruction water versus land exercise. However, during this time it was not shown that training in the aquatic environment could be as effective in older adults as land exercise in order to improve one's physical condition. Until the past decade, no research groups considered that the limitation of the aquatic environment was due to the lack of control of the training load during the exercise. The year 2005 is estimated as an ideal date to begin reviewing literature as before this time, the scientific rigor of relevant studies was low as they were directed solely for therapeutic exercise such as knee injuries or hip osteoarthritis [21]. From studies where the intensity of the exercise is controlled in a systematic

Table 1 Search Strategy

\section{Search Strategy}

The terms "aquatic-exercise" ("water-based training", "water exercise", "hydrotherapy", "exercise-aquatic) and "fall" ("fall-prevention", "fall risk") were combined with the terms: "older-adults", "elderly", "aging", "physical-exercise", "physical-fitness", "balance", "strength" and "muscle mass". 
way through adapted material, depth, type of exercise and number of series, aquatic exercise can be better understood by healthcare professionals [22].

\section{Study population}

The study population consisted of healthy adults aged between 60 and 80 years old.

\section{Type of intervention and comparisons}

To be included within the study, the articles were required to state the training methodology adopted within an aquatic environment. Studies were also included if they compared either (i) aquatic exercise and non-aquatic exercise, or (ii) aquatic exercise and aquatic exercise with different types of intervention. Studies that did not report their intervention methodology to prevent falls were excluded.

\section{Type of outcomes}

Assessments of physical function and/or physical performance based on fall prevention in an aquatic environment were the outcomes in the included studies. Data were extracted which related to outcomes about the intensity, volume, type of exercise, frequency and recovery.

The studies retrieved were assessed against the inclusion criteria, based on their titles. The full text of the remaining studies was then independently reviewed by two researchers (EMCG and LB). An inclusion and exclusion criteria can be found in Table 2.

\section{Data collection process, data items and summary measures}

Data were extracted by one reviewer (EMCG) using a standardized template and verified by a second reviewer (JO). Information was extracted from each included trial on: authors, year of publication, study design, population characteristics (number of participants, gender and age), description of intervention and outcome measures. Disagreements or discrepancies on data extraction were resolved by discussion.

\section{Methodological quality in individual studies}

The methodological quality of all studies was assessed using the PEDro (Physiotherapy Evidence Database) appraisal instrument [23]. The 11 item scale is a valid and replicable measure used to critically appraise the quality of randomized controlled trials. Each study is scored out of ten, with a threshold of seven (or over) for a study to be considered high quality (item one on the scale indicates external validity).

\section{Results}

A summary of the selection process is schematized in Fig. 1. The literature search yielded a total of 143 articles, of which 12 were duplicates and 81 were immediately excluded after reviewing the title, as they did not fit the inclusion criteria. A total of 40 full-text articles were reviewed. Thirty-two articles could be obtained through the search engine of the Catholic University of Murcia and its corresponding subscriptions to certain journals. The eight remaining articles were obtained on free access web pages or through an interlibrary loan process. Following a full text review (EMCG and TI), fourteen studies were included in the synthesis of results.

Twelve of the sourced studies were randomized controlled trials (RCTs) [16, 24-34] and two studies adopted a quasi-experimental design $[35,36]$. Nine studies specifically evaluated the impact of aquatic training on improving strength $[16,25,27,29-32,35,36]$, nine studies assessed balance following aquatic training [24, 26, 28 $30,32,33,35,36]$, and four studies compared a variety of physical fitness components, including agility, endurance, flexibility and speed $[26,27,35,36]$.

Table 2 Eligibility criteria

\begin{tabular}{|c|c|}
\hline Inclusion Criteria & Exclusion Criteria \\
\hline \multicolumn{2}{|l|}{ Population } \\
\hline Healthy adults & Adults with pathologies or comorbidities \\
\hline \multicolumn{2}{|l|}{ Aged between 60 and 90} \\
\hline \multicolumn{2}{|l|}{ Interventions } \\
\hline Studies that directly or indirectly assess the prevention of falls in an aquatic environment & Methodology not clearly stated \\
\hline \multicolumn{2}{|l|}{ Outcome Measures } \\
\hline \multicolumn{2}{|l|}{ Physical function } \\
\hline \multicolumn{2}{|l|}{ Physical performance based on fall prevention } \\
\hline \multicolumn{2}{|l|}{ Methodology } \\
\hline Randomised or non-randomised clinical trial. & Reviews, study protocols or case studies. \\
\hline \multicolumn{2}{|l|}{ Publication } \\
\hline Published between 2005 and 2018 & Published before 2005 \\
\hline
\end{tabular}




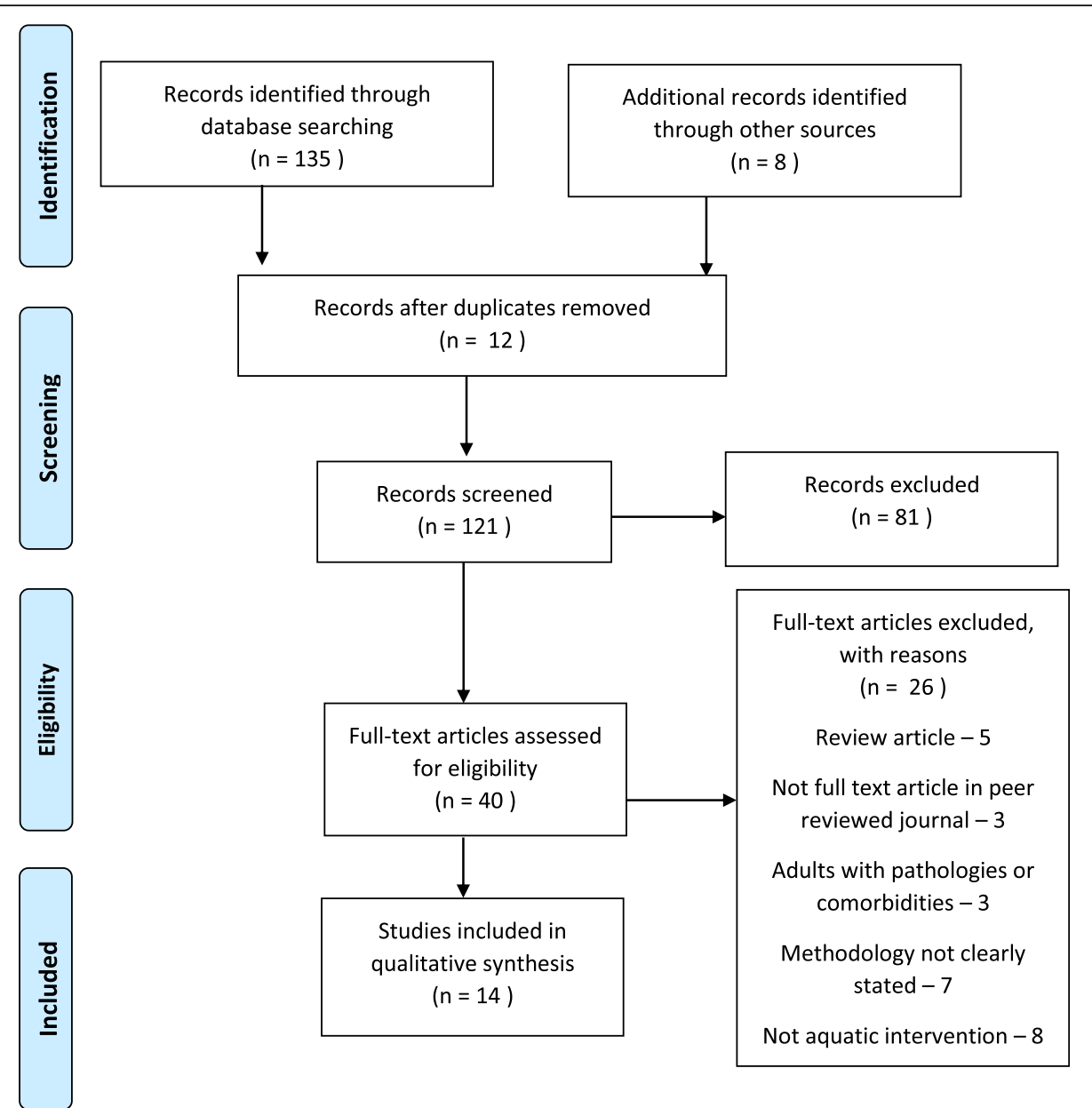

Fig. 1 Flow chart illustrating each phase of study selection

\section{Measuring instruments}

The flexibility variable was recorded by the chair sit-and-reach and the back scratch test in one study $[26,30]$ whilst another study used the functional tests of Johnson and Nelson [35, 36]. The remaining studies utilised the sit and reach test [25, 27, 29].

Dynamic balance was measured using the berg balance scale [33], the timed up and go test [24, 25, 27, 28, 35], the $8 \mathrm{ft}$ up and go test $[29,30,34]$ and the walking in circle test [36]. Static balance was recorded with the Sharpened Romberg test $[24,28,35]$ with both open and closed eyes, it was also registered by a Biodex Balance Stability System [32] and using a force platform where the subjects kept their arms on their hips to maintain position [36].

The variable of strength was predominantly recorded for upper limbs using a hand dynamometer [25, 27, 30, 32]. The maximal dynamic strength was evaluated using the 1-repetition maximum (1RM) bench press [16] bilateral elbow flexion [16, 26, 36] and bilateral knee flexion/extension [16]. The muscular endurance was evaluated during a bilateral knee extension and flexion with a load equivalent to $60 \%$ of $1 \mathrm{RM}$ maintaining a maximum possible number of repetitions [16]. An isokinetic machine was also used for lower limbs. In this case a knee flexion-extension is performed at speeds of $60 \% \mathrm{~s}, 120 \% \mathrm{~s}$ [26]. In addition, a functional test like the $30 \mathrm{~s}$ sit-and-stand-up were used by a large number of researchers [26, 29, 31, 36].

Flexibility was mainly measured by the sit-and-reach test for the hamstrings muscles and lower back [25-27, $29,30]$ and the back-scratch test was used to assess the general shoulder range of motion $[26,30]$.

\section{Study population}

When divided into their respective experimental groups, nine of the studies sourced had sample sizes of between 13 and 17 subjects $[16,24-27,30-33,35]$. The remaining studies tested between 20 and 66 subjects [28, $29,34,36]$. Four studies assessed both male and female participants [27-30], four studies only included women $[16,25,31,36]$ and three studies only assessed male participants [24, 32, 35]. The age range of the study 
populations was generally small, however in four studies the range was 15 years $[16,25,29,35]$ and in two studies the range was over 24 years [33, 36].

\section{Intervention variables}

The temperature of the water varied between $28^{\circ} \mathrm{C}$ and 32 ${ }^{\circ} \mathrm{C}$ within the majority of studies, $[16,25-27,29,34-36]$ with the exception of four studies, [24, 28, 30,31] where the temperature was over $32{ }^{\circ} \mathrm{C}$ and two studies which do not specify the water temperature $[32,33]$. The depth of the water in most of the interventions was from between waist to the midline of the chest $[24,26,28]$ and at the level of the xiphoid process $[16,25,29-31,33-36]$ except in two studies $[30,32]$ where the depth reached $1.80 \mathrm{~m}$. The shorter studies had a duration of two weeks [28] and six weeks $[24,30]$. Most interventions were implemented for eight to 12 weeks $[16,26,27,29,31-35]$. However, one study completed a 16 weeks intervention [36], and the longest intervention had a duration of 24 weeks [25]. The frequency of the intervention was generally between two sessions $[16,24,30,31,33,35,36]$ and three sessions a week [25-27, 29, 32, 34] although there is a study with a frequency of 5 days a week for 2 weeks during the intervention [28]. In terms of duration, most of the interventions were $60 \mathrm{~min}$ long, [25, 26, 28-30,32, 35, 36] however, one study had a duration of $90 \mathrm{~min}$ [28], another of $40 \mathrm{~min}$ [34] and four studies did not specify the duration of the intervention $[17,25,32,35]$.

In some cases, the intensity of training was described by referring to the Borg Rate of Perceived Exertion Scale, the same Borg scale which ranges from six to 20 points. A value of one represents a very light exercise and 20 a serious physical exertion [37]. Moderate to high intensity was mentioned in ten of the included results [16, 25-27, $29-31,34-36]$ and exercise intensity was not systematically recorded in the remaining studies [24, 28, 32, 33].

\section{Physiological improvements}

The control group, absent from any intervention, did not obtain any significant difference at the end of the study $[25,26,28,30-36]$. However, the aquatic intervention group obtained improvements $(p \leq 0.05)$ at the end of the study in the variable of flexibility $[25-27,29,30,36]$, balance $[24,26,28,32,35,36]$ and strength [16, 25-27, 29-32, 36].

Regarding the four studies that include a third exercise programme in a land environment, two of them did not find differences between the aquatic and land groups after the intervention, in variables of flexibility, balance and strength [24, 28]. Nevertheless, the aquatic group obtained greater improvement over the terrestrial group in the variable of balance in one study [30]. Another study [27] compared two aquatic groups with different types of intervention: resistance material and without any material. The material group presented better results in gait speed, however, in variables of strength, mobility and flexibility, no significant differences were found between groups.

\section{Resistance material}

The studies did not specify what kind of materials they used except in one case [27] where the aquatic group used newly made water-resistance equipment (power leg and power hand DESCENTE Co., Ltd., Japan) and another study [36] where the aquatic group progressed at their own rate by adding surface area equipment (Aqua Flex paddles by MIZUNO Corporation, Osaka, Japan) and by opening the webbing of the Aqua Mitt gloves to increase surface area to reach the goal RPE.

\section{Type of exercise}

The studies that introduce flexibility training do not indicate specific exercises that are performed [26, 27, 29]. Only five studies indicate the static and dynamic balancing exercises which they use $[26,28,33,35,36]$. When looking at strength training there is a general focus on large muscle groups from the upper or lower limbs [16, $24,26,27,31,32,34]$. Only two studies [31, 36] mention the execution of exercises that resemble activities of daily living (ADL). A schematic compression can be viewed in Table 3.

\section{Discussion}

There is evidence to support the use of aquatic exercise in order to modify variables related to the risk of fall in the elderly. Aquatic exercise may ameliorate the negative physiological effects of aging which are predisposing, modifiable risk factors of a fall. Improving these risk factors is likely to create an overall benefit to rate of falls, and thus aquatic exercise should be considered as a training method for those most at risk of falling. However, the relationship between aquatic exercise and fall prevention is not directly compared within the studies sourced.

\section{Balance}

Studies which assessed balance within their training programmes found significant improvements $(p \leq 0.05)$ following the intervention [24, 28, 32, 34-36] with the exception of two studies [26,33]. The lack of positive results in balance training may be due to the lack of scientific rigor and the specificity of training. In this review, the exercises used most for balance training are alternating movements of the upper and lower limb [24, 26, 28, $33,35,36]$ however, their training variables such as the control of intensity or the specific exercises are not clearly reported in the methodology. Balance is considered the most important variable during the design of a 


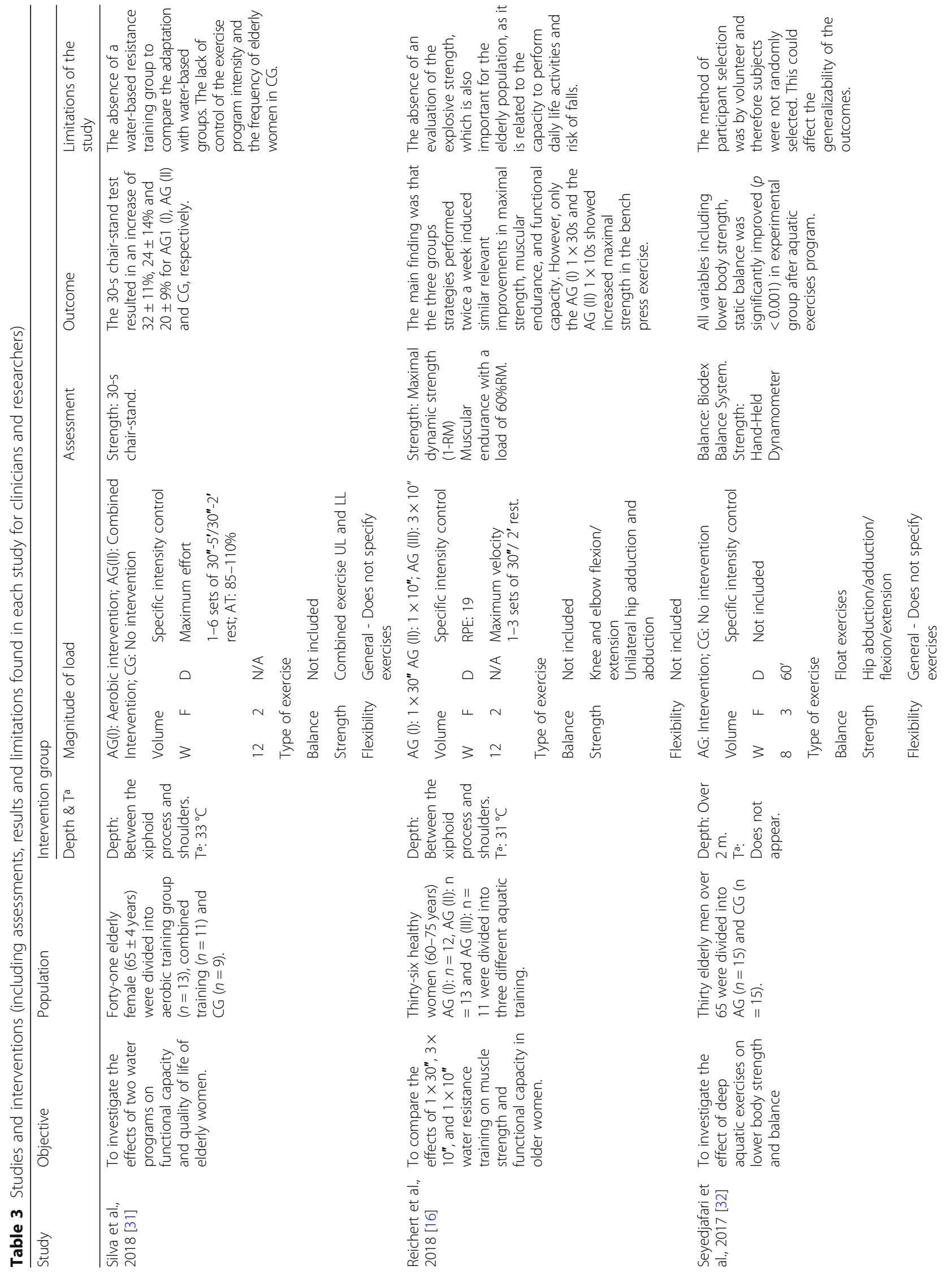




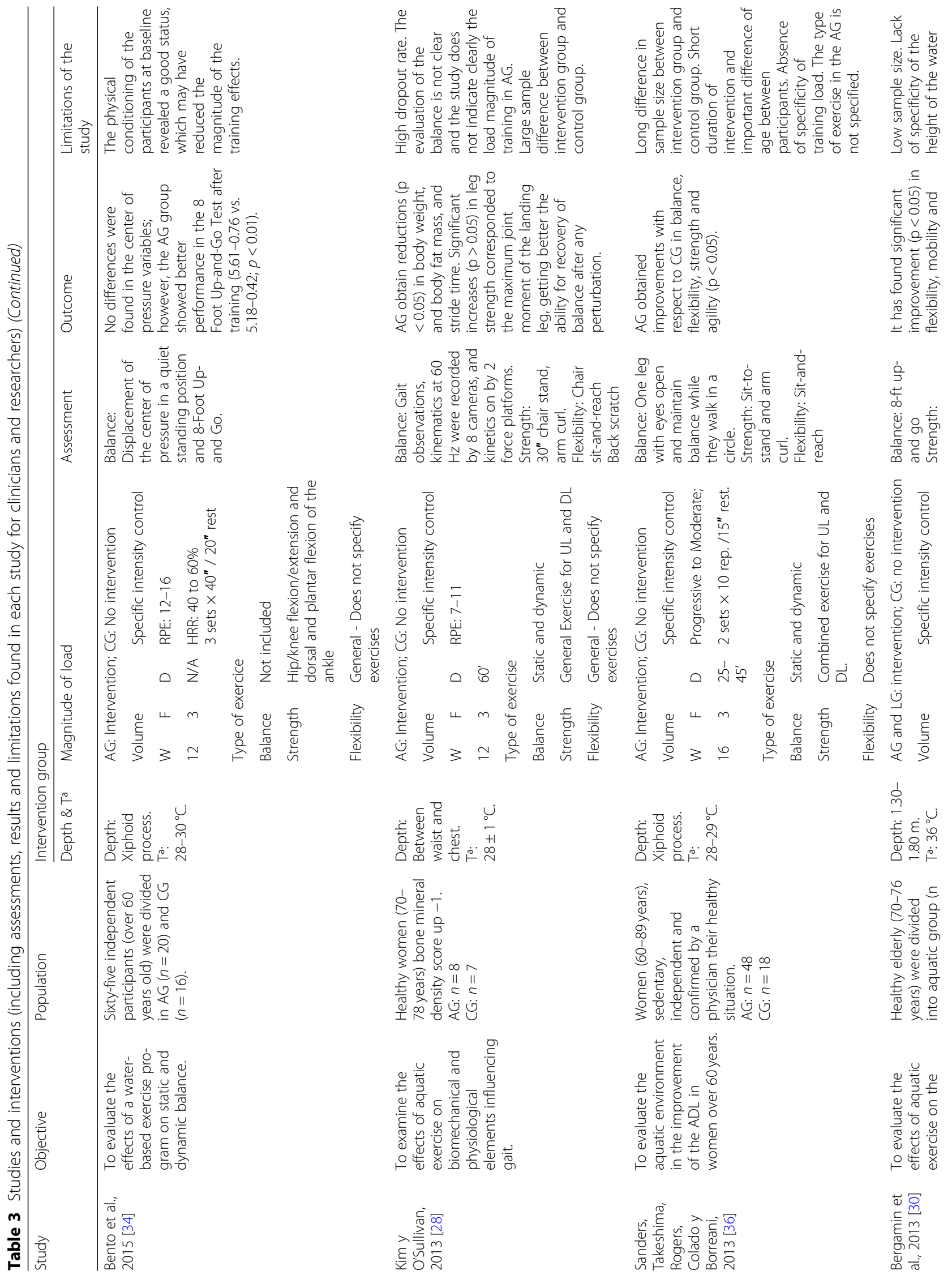




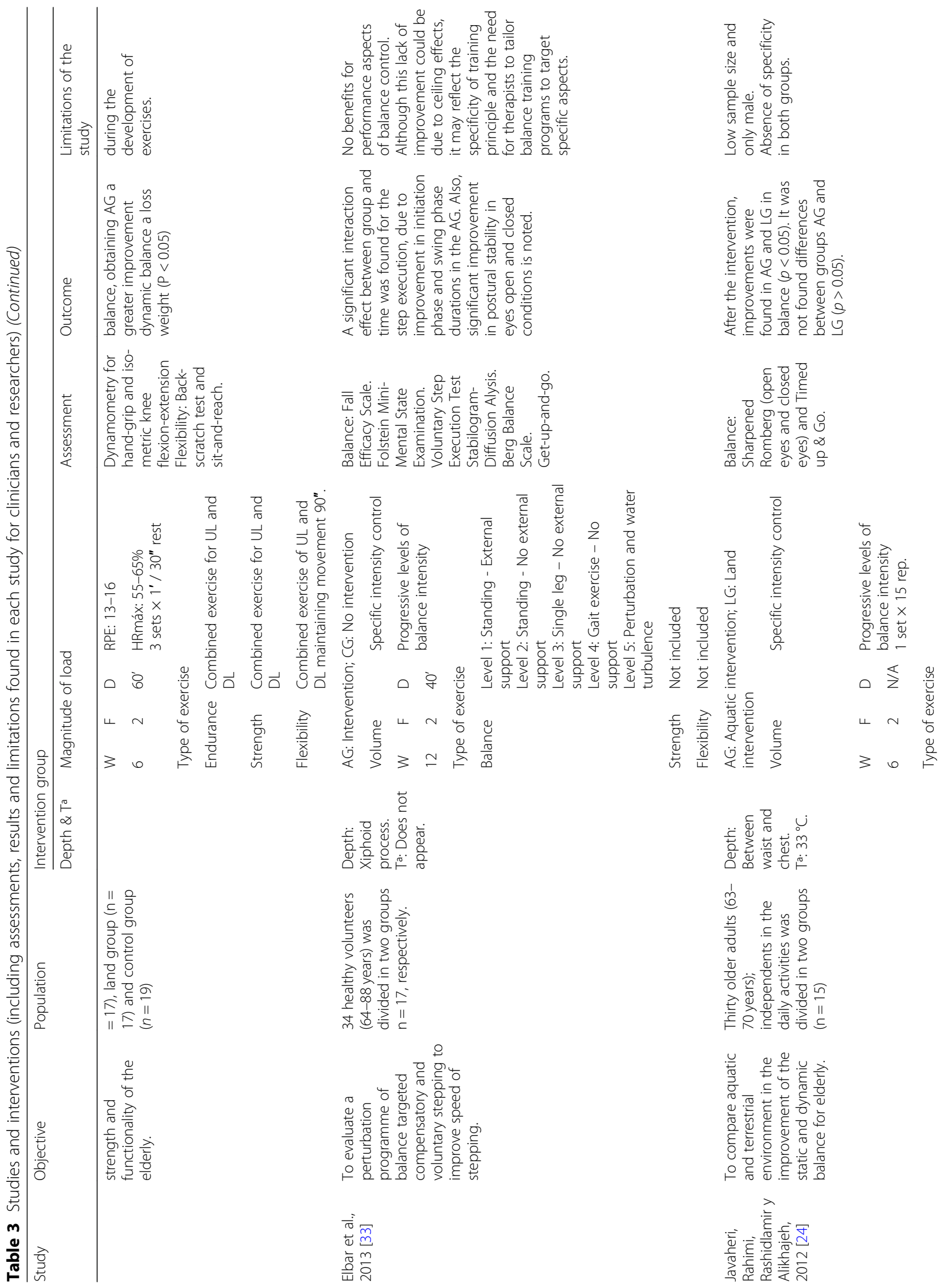




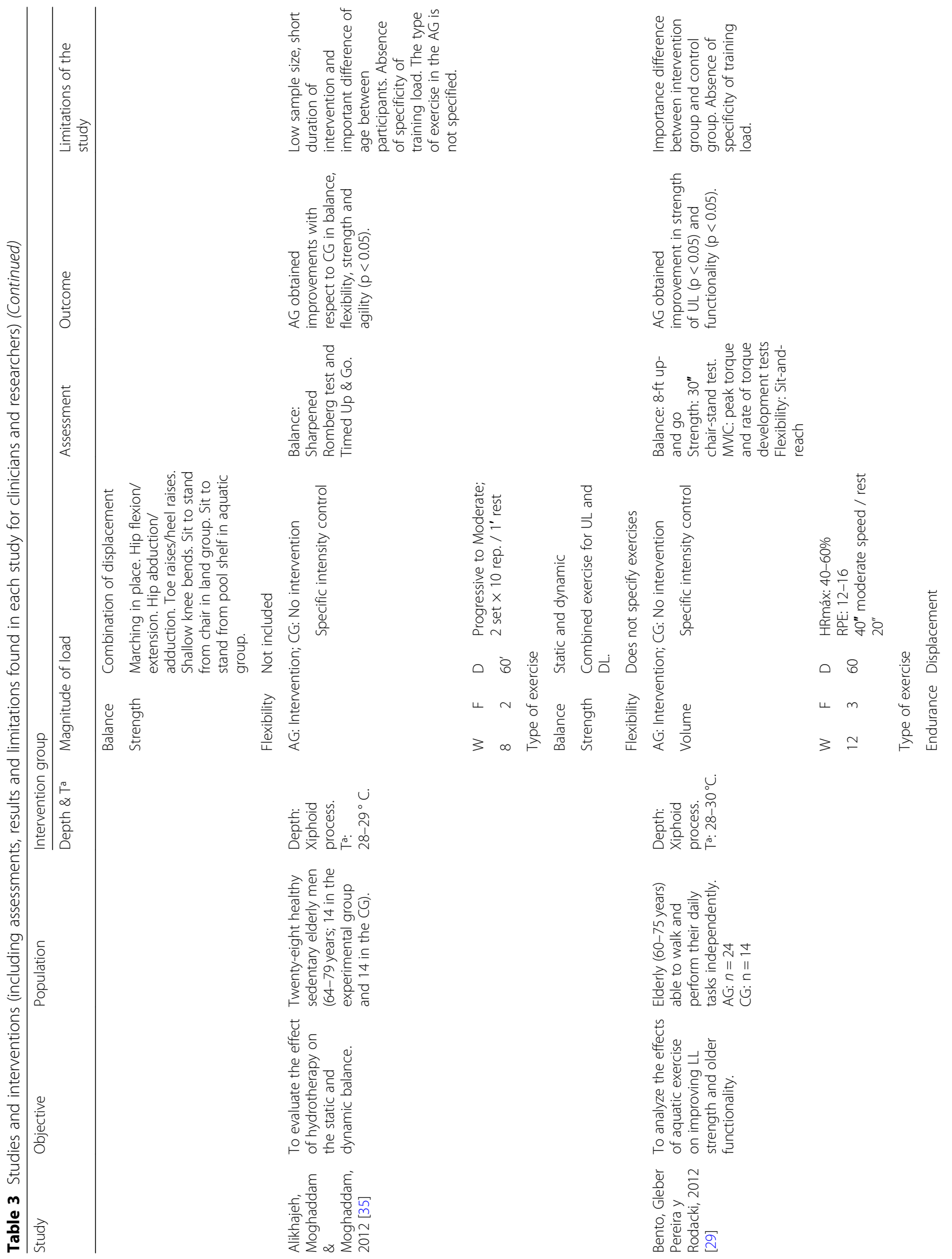




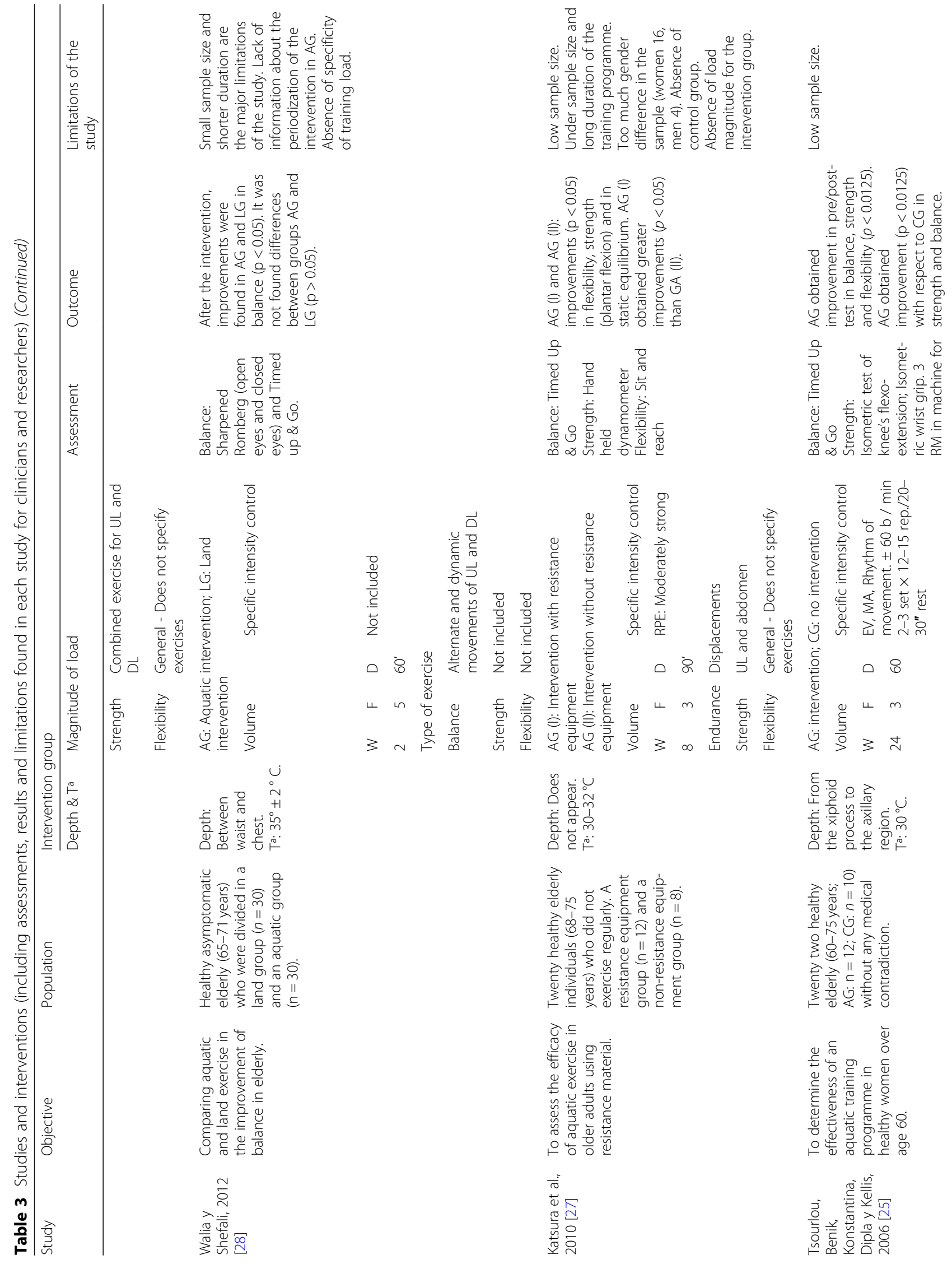




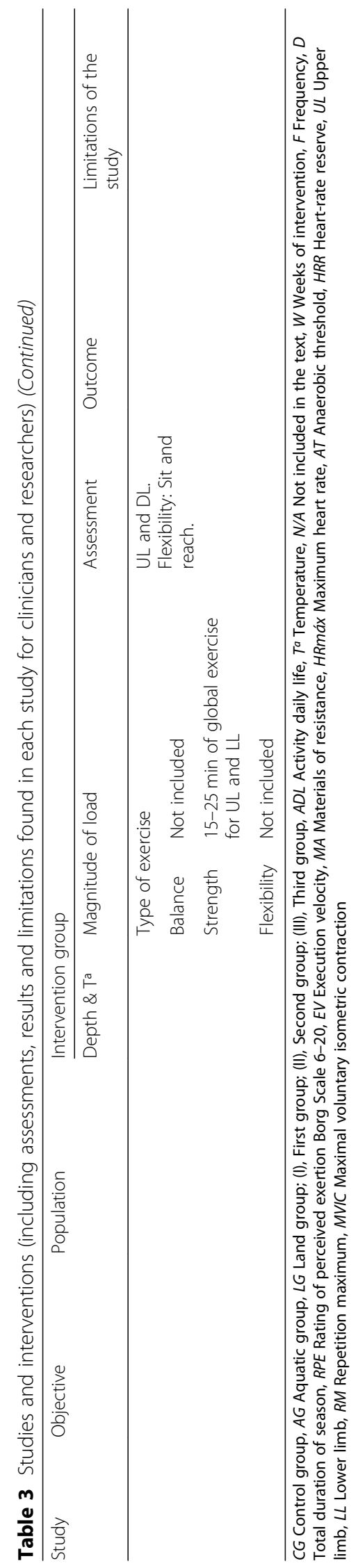


fall prevention training programme [38], and consequently the specificity of the exercises and the control of training should appear in order to reproduce the results obtained in the studies. Therefore, certain criteria or principles that support the decisions made during the fall prevention program should be considered during the design of protocols to improve balance in older people. An example are those established by two experts $[8,38]$ : the reduction of the support base, the movement of the centre of gravity, the mobilization of the limbs and the reduction of the visual field $[8,38]$. It has been recommended that due to the aquatic turbulence during the aquatic exercise, muscle are activated continuously to stabilize the position of the individual [16]. However, the depth of the water and velocity of exercise should be controlled in order to modify muscle activation during balance exercises [39].

One study [36] demonstrated that in a group of 66 subjects (60-89 years), during a 16-week programme which included strength training, in an elderly population it is possible to achieve a better static balance in the elderly. A similar study [24] evaluated the static and dynamic balance in two groups of 15 subjects (63-70 years) during two equally designed training programmes in both a land and aquatic environment. At six weeks, both groups achieved significant improvements in static and dynamic balance $(p<0.05)$ when compared to pre-test scores. Similarly, a significant gain $(\mathrm{p}<0.05)$ was found for the Berg Balance Score in adults older than 68 years old with no significant difference from one environment to another $(p>0.05)$.

Although the studies reviewed did not find significant differences between land and water training, the results suggest that an aquatic environment offers a safe environment where the risk of falling is eliminated. It should, however, be noted that there is evidence to highlight the insufficiency of the Berg Balance Scale for predicting falls [40] and the Timed Up and Go Test is instead recommended by the U.S. Centres for Disease Control and Prevention as an easy to administer clinical test to evaluate a senior citizen's fall risk [41].

One study assessed the effect of aquatic training on agility [20]. The authors found significant improvements in the intervention group receiving an aquatic exercise programme in comparison to the control group $(p<0.05)$. There is evidence that agility training programmes can reduce the risk of falls [42], largely due to improved balance, which has been examined as a variable for improving mobility in older adults. A study comparing fall prevention programmes in older adults [43] found that visual training had the greatest improvement on obstacle course performance $(22 \%)$ following a 12 -week training program. In addition, agility has been found to reduce the risk of falling through step training [44], and in multifactorial programs which focus on other variables such as balance and strength [45].

\section{Strength}

Aquatic interventions that trained large muscle groups of the upper and lower limbs found significant improvements in strength from pre-test to post-test $(p<0.05)$ $[16,25-27,29-32,36]$. Although the methodologies of these protocols vary, all results indicate that an aquatic environment is beneficial for promoting muscle hypertrophy. Strength training cause changes in the nervous system through neuromuscular activation, resulting in improved muscular power and delayed onset of sarcopenia which may decrease the risk of falls [46]. Evidence for the effectiveness of lower limb strengthening exercises for the prevention of falls in elderly subjects has been previously reported [47-49] and as lower extremity weakness is a clinically significant risk factor of a fall. The design of aquatic exercise programmes should focus on the lower limbs, respecting the principle of training specificity. Lower limb weakness in older adults is considered an important influencing factor of falls and its preservation promotes the maintenance of independence. More specifically, the maximum voluntary isometric strength and the rate of force generation of the hip abductors are considered a useful measure to distinguish between older adult fallers and non-fallers [50]. However, several studies use the handgrip dynamometer as a measure for strength in the elderly [25, 27, 32]. Although this outcome measure does not specifically assess lower limb strength, a recent study developed by the Cooperative Health Research in the Region of Augsburg [51] with 808 individuals (age $\geq 65$ years) found a trend toward an indirect effect of grip strength on balance problems $(p$ value $=.043)$. In addition, handgrip strength has been estimated by other studies as an Indicator of Health-Related Quality of Life in Old Age and fall prevention $[52,53]$.

\section{Flexibility}

Axial stiffness can occur through ageing and is a loss of flexibility and elasticity in the neck and trunk that causes the imbalance of the posture, thus reducing balance and increasing the chance of falling. The ACSM (American College of Sports Medicine) [47] highlights the importance of flexibility training during aging for fall prevention; however often it remains neglected in training programmes.

Within this review, six studies assessed flexibility as an outcome following aquatic training $[23,25,27,29,30,36]$. In all cases, flexibility was seen to improve significantly from pre-test to post-test $(p \leq 0.05)$, however the specific effect on each muscle group was not clearly described. Regarding the principle of specificity and the relationship of 
flexibility with the risk of falls, the findings indicate that the rigidity in the pelvic muscles causes an alteration in the gait of the elderly, which will contribute to an increased risk of falls [54]. In this case, a specific program of hip flexor stretches is needed, which may reverse variables that increase the risk of falls in the elderly [55].

\section{Duration}

Six studies [16, 26, 29, 31, 33, 34] agree with others found in the literature [56-58] that a training programme that seeks to allow for significant changes in balance, strength or flexibility to prevent falls in older adults should be a minimum duration of 12 weeks. To make a positive impact, the duration of the session should be around $60 \mathrm{~min}$ and two to three times a week $[16,26,29,31-34]$, as recommended by similar studies $[48,57,59,60]$. The ACSM expresses an optimum frequency of three times a week at a moderate-to-high intensity to facilitate fall prevention training in the elderly [47]. This exercise prescription allows for physical recovery that induces physiological adaptations (48-72 $\mathrm{h}$ between sessions) [61]. However, studies with less frequency and duration also found significant improvements in balance, strength and flexibility tests [24, $27,30,32,35,36]$, after their respective interventions. These results indicate that the intensity of a programme will be a greater modifier of effect than the volume of training to reach physiological adaptations in the elderly and to improve functional capacity $[16,62,63]$. Therefore, it seems likely that frequency, duration and intensity of exercises, including those related to strength, balance, and flexibility are relevant training principles for reducing the risk of falls in the elderly.

\section{Intensity}

The intensity of an exercise programme is an important factor for determining the physiological stress and subsequent adaption an individual can experience through training. If the frequency and intensity of the training programme is too high, muscular damage may occur and this can negatively affect adherence to programmes [64]. The Borg Scale is a valid and simple measure of controlling intensity that has been utilised within the studies analysed [16, 26, 27, 29, 30, 34, 36]. In addition, using Rate of Perceived Exertion (RPE) [37] to prescribe exercise intensity is considered appropriate for older adults $[65,66]$. However, in many cases, the number of sets, repetitions or recovery periods was missing from the methodology, preventing the protocols being repeated. Only one study [30] explained the control of the intensity of the flexibility whereby the involved muscles were contracting for 60-90 s during stretching.

Intensity should be controlled like with land exercise but with consideration of the principles of hydrodynamics and the forces that influence aquatic exercise to improve physiological responses in older adults [67]. For example, in one study [17], three different types of training were compared over ten weeks: land training with machines $(n=14)$, training with elastic bands $(n=21)$ and water resistance training $(n=17)$ following the same protocol training but adapted to the environment. The three groups obtained significant differences $(p<0.05)$ in flexion of arms (push up), squats, crunches and in the fat-free mass. It is shown that controlled resistance training in the aquatic environment can be as beneficial for the improvement of physical condition and body composition as land training when the protocol is adapted to the specific nature of the environment. In this way, a table of the intervention of the studies is designed, which will serve for clinical protocols and researchers to know the variables of exercise prescription of each of the articles analysed (Table $3)$.

\section{Methodology quality}

Generally, the methodological quality of the studies included was low. The mean PEDro score for all fourteen studies was 5.5/10 (Table 4). A score of seven (or over) is considered high quality [68], and therefore only on studies in this review had a high quality methodology [33]. Thirteen studies were considered 'low quality' when using a threshold of six or less out of ten [16, 24-32, 34-36]. Due to the nature some interventions, it was difficult to blind subjects, therapists and assessors and therefore consistently low scoring items on the scale were: three, five, six and seven.

It is difficult to advocate the use of one study over another due to a lack of repeated designs. This makes it difficult to establish a "fixed criteria" in the development of training programmes in the aquatic environment in order to reduce the risk of falls in the elderly.

Although the studies mention the reduction of fall risk with the results obtained, they do not present correlations between variables of strength, balance or flexibility that could suggest a strong clinical relationship. Well-written methodologies should express all relevant information on the training design (volume, intensity, frequency, recovery and type of exercise) in a clear and replicable way. They should also present correlations between falls and physical training variables such as agility, balance, strength and flexibility.

\section{Study limitations}

There is some evidence to support using aquatic exercise for improving predisposing, modifiable risk factors of falls. However, the quality of this evidence is low, and many interventions are poorly described. The lack of consistency between study methodologies makes intervention comparison difficult. In addition, the trial findings did not find a statistically significant relationship 
Table 4 Grade of evidence PEDro score

\begin{tabular}{|c|c|c|c|c|c|c|c|c|c|c|c|c|c|}
\hline \multirow[t]{2}{*}{ Study } & \multirow{2}{*}{$\begin{array}{l}\text { PEDro Score } \\
\text { out of } 11\end{array}$} & \multicolumn{11}{|c|}{ Item } & \multirow{2}{*}{$\begin{array}{l}\text { Sample } \\
\text { size }\end{array}$} \\
\hline & & 1 & 2 & 3 & 4 & 5 & 6 & 7 & 8 & 9 & 10 & 11 & \\
\hline Silva et al., 2018 [31] & $6 / 10$ & $\cdot$ & $\cdot$ & & $\cdot$ & & & $\cdot$ & & $\cdot$ & $\cdot$ & $\cdot$ & 41 \\
\hline Reichert et al., 2018 [16] & $6 / 10$ & $\cdot$ & $\cdot$ & & $\cdot$ & & & $\cdot$ & & $\cdot$ & $\cdot$ & $\cdot$ & 36 \\
\hline Seyedjafari et al., 2017 [32] & $4 / 10$ & $\cdot$ & & & - & & & & & - & · & · & 30 \\
\hline Bento et al., 2015 [34] & $5 / 10$ & $\cdot$ & $\cdot$ & & $\cdot$ & & & & & $\cdot$ & $\cdot$ & $\cdot$ & 36 \\
\hline Kim y O'sullivan, 2013 [28] & $5 / 10$ & $\cdot$ & $\cdot$ & & $\cdot$ & & & & & $\cdot$ & $\cdot$ & $\cdot$ & 15 \\
\hline Sanders et al., 2013 [36] & $5 / 10$ & $\cdot$ & & & $\cdot$ & & & & $\cdot$ & $\cdot$ & $\cdot$ & $\cdot$ & 60 \\
\hline Bergamin et al., 2013 [30] & $6 / 10$ & $\cdot$ & $\cdot$ & & $\cdot$ & & & & $\cdot$ & $\cdot$ & $\cdot$ & $\cdot$ & 53 \\
\hline Elbar et al., 2013 [33] & $8 / 10$ & $\cdot$ & $\cdot$ & $\cdot$ & . & & & $\cdot$ & . & - & · & . & 34 \\
\hline Javaheri et al., 2012 [24] & $6 / 10$ & $\cdot$ & $\cdot$ & & $\cdot$ & & & & $\cdot$ & $\cdot$ & $\cdot$ & $\cdot$ & 28 \\
\hline Alikhajeh et al., 2012 [35] & $4 / 10$ & $\cdot$ & & & & & & & $\cdot$ & $\cdot$ & $\cdot$ & $\cdot$ & 28 \\
\hline Bento et al., 2012 [29] & $5 / 10$ & $\cdot$ & $\cdot$ & & $\cdot$ & & & & & $\cdot$ & $\cdot$ & $\cdot$ & 38 \\
\hline Walia y Shefali, 2012 [28] & $6 / 10$ & $\cdot$ & $\cdot$ & & $\cdot$ & & & & $\cdot$ & $\cdot$ & $\cdot$ & $\cdot$ & 60 \\
\hline Katsura et al., 2010 [27] & $5 / 10$ & $\cdot$ & $\cdot$ & & & & & & $\cdot$ & $\cdot$ & $\cdot$ & $\cdot$ & 20 \\
\hline Tsourlou et al., 2006 [25] & $6 / 10$ & $\cdot$ & $\cdot$ & & $\cdot$ & & & & $\cdot$ & $\cdot$ & $\cdot$ & $\cdot$ & 22 \\
\hline
\end{tabular}

- $=$ Met this criterion OF PEDro Scale

between training variables and falls. Resistance equipment is not included to increase exercise intensity, and few studies highlight the importance of execution speed during the aquatic exercises. Regarding the types of exercise, most of them refer to general exercises of upper and lower limbs; however, the exercises should be described in detail so that professionals can achieve the desired results. Regarding the flexibility variable, it is included during the programs directed to older adults but it is not discussed as a relevant variable. Flexibility is not treated in a specific way, obviating the importance of the pelvic musculature in the disturbance of the gait and therefore of the risk of falling. Researchers should avoid describing a superficial and uncritical methodology in order to design specific and valid aquatic programs for reducing the most modifiable predisposing risk factors of falls within the elderly.

\section{Conclusions}

There is some evidence to support using aquatic exercise for improving predisposing, modifiable risk factors of falls. However, the quality of this evidence is low, and many interventions are not fully described. The lack of consistency between study methodologies makes intervention comparison difficult. In addition, the trial findings did not find a statistically significant relationship between training variables and falls.

Despite the inconsistent and lacking results, it should be noted that there were no detrimental or counterproductive effects found within the search results following a physical aquatic exercise programme. With increased consistency in the design of evidence-based training programmes, the aquatic environment could become widespread and effective for helping to prevent the risk of falls in the elderly. Further research is needed to create an evidence-based, replicable protocol for aquatic training with a specific aim of improving the commonly reported predisposing risk factors of falls.

\section{Abbreviations}

ACSM: American College of Sports Medicine; ADL: Activity of Daily Living: PRISMA: Preferred Reporting Items for Systematic Reviews and Meta-Analyses

\section{Acknowledgements}

Not applicable.

Funding

No funding was received for this study.

Availability of data and materials

The materials used within this study were taken directly from the research articles which were sourced from our literature search. We can provide copies of this data if required.

\section{Authors' contributions}

EMCG: Developing design, literature search, manuscript writing, and analysis of results. LB: Manuscript writing and developing design. TI: Quality assessment. TW: Supervision of data collection and analysis. AMAA: Supervision of developing design and literature search. All authors read and approved the final manuscript.

Ethics approval and consent to participate Not applicable.

Consent for publication

Not Applicable.

Competing interests

The authors declare that they have no competing interests. 


\section{Publisher's Note}

Springer Nature remains neutral with regard to jurisdictional claims in published maps and institutional affiliations.

Received: 10 January 2018 Accepted: 13 February 2019

Published online: 22 February 2019

\section{References}

1. World Health Organization. Global report on falls prevention in older age. http://www.who.int/ageing/publications/Falls_prevention7March.pdf.

2. Society AG, Society G, Prevention OF, Panel OS. Guideline for the prevention of falls in older persons. J Am Geriatr Soc. 2001;49(5):664-72.

3. Tinetti ME, Speechley M. Prevention of falls among the elderly. N Engl J Med. 1989;320(16):1055-9.

4. da Silva Gama ZA, Gómez-Conesa A. Factores de riesgo de caídas en ancianos: revisión sistemática. Rev Saude Publica. 2008;42(5):946-56.

5. Scuffham P, Chaplin S, Legood R. Incidence and costs of unintentional falls in older people in the United Kingdom. J Epidemiol Community Health. 2003;57(9):740-4

6. Pernambuco CS, Borba-Pinheiro CJ, de Souza Vale RG, Di Masi F, Monteiro PKP, Dantas EHM. Functional autonomy, bone mineral density (BMD) and serum osteocalcin levels in older female participants of an aquatic exercise program (AAG). Arch Gerontol Geriatr. 2013;56(3):466-71.

7. Walston JD. Sarcopenia in older adults. Curr Opin Rheumatol. 2012;24(6):623.

8. Rubenstein LZ. Falls in older people: epidemiology, risk factors and strategies for prevention. Age Ageing. 2006;35(suppl_2):ii37-41.

9. Moreland JD, Richardson JA, Goldsmith $\mathrm{CH}$, Clase CM. Muscle weakness and falls in older adults: a systematic review and meta-analysis. J Am Geriatr Soc. 2004;52(7):1121-9.

10. Myers AH, Young $Y$, Langlois JA. Prevention of falls in the elderly. Bone. 1996;18(1):S87-S101.

11. Russell K, Taing D, Roy J. Measurement of fall prevention awareness and behaviours among older adults at home. Can J Aging. 2017;36(4):522-35.

12. El-Khoury F, Cassou B, Charles M-A, Dargent-Molina P. The effect of fall prevention exercise programmes on fall induced injuries in community dwelling older adults: systematic review and meta-analysis of randomised controlled trials; 2013.

13. Scapagnini G, Caruso C, Spera G. Preventive medicine and healthy longevity: Basis for sustainable anti-aging strategies. In: International Textbook of Aesthetic Surgery. Springer; 2016. p. 1213-27. https://link. springer.com/chapter/10.1007/978-3-662-46599-8 82

14. Lees FD, Clark PG, Nigg CR, Newman P. Barriers to exercise behavior among older adults: a focus-group study. J Aging Phys Act. 2005;13(1):23-33.

15. Simas V, Hing W, Pope R, Climstein M. Effects of water-based exercise on bone health of middle-aged and older adults: a systematic review and meta-analysis. Open Access J Sports Med. 2017;8:39.

16. Reichert T, Delevatti RS, Prado AKG, Bagatini NC, Simmer NM, Meinerz AP Barroso BM, Costa RR, Kanitz AC, Kruel LFM. Low-and High-Volume WaterBased Resistance Training Induces Similar Strength and Functional Capacity Improvements in Older Women: A Randomized Study. J Physical Activity Health. 2018;20(XX):1-8.

17. Colado J, Garcia-Masso X, Rogers M, Tella V, Benavent J, Dantas E. Effects of aquatic and dry land resistance training devices on body composition and physical capacity in postmenopausal women. J Human Kinetics. 2012;32: 185-95.

18. Moher D, Liberati A, Tetzlaff J, Altman DG, Prisma G. Preferred reporting items for systematic reviews and meta-analyses: the PRISMA statement. PLoS Med. 2009;6(7):e1000097.

19. Urrútia G, Bonfill X. Declaración PRISMA: una propuesta para mejorar la publicación de revisiones sistemáticas y metaanálisis. Med Clin. 2010; 135(11):507-11.

20. Tovin BJ, Wolf SL, Greenfield BH, Crouse J, Woodfin BA. Comparison of the effects of exercise in water and on land on the rehabilitation of patients with intra-articular anterior cruciate ligament reconstructions. Phys Ther. 1994:74(8):710-9.

21. Crawford MT. Aquatic Exercise For Rehabilitation And Training. J Orthopaedic Sports Physical. 2011:41(4):286-7.

22. Colado JC, Borreani S, Pinto SS, Tella V, Martin F, Flandez J, Kruel LF. Neuromuscular responses during aquatic resistance exercise with different devices and depths. J Strength Cond Res. 2013;27(12):3384-90.
23. Maher CG, Sherrington C, Herbert RD, Moseley AM, Elkins M. Reliability of the PEDro scale for rating quality of randomized controlled trials. Phys Ther. 2003;83(8):713-21.

24. Hashemi Javaheri SAA, Mohammad Rahimi N, Rashidlamir A, Alikhajeh Y.The effects of water and land exercise programs in static and dynamic balance among elderly men. Global J Guid Couns. 2012;2(1):1-7.

25. Tsourlou T, Benik A, Dipla K, Zafeiridis A, Kellis S. The effects of a twentyfour-week aquatic training program on muscular strength performance in healthy elderly women. J Strength Cond Res. 2006;20(4):811.

26. Kim SB, O'sullivan DM. Effects of aqua aerobic therapy exercise for older adults on muscular strength, agility and balance to prevent falling during gait. J Phys Ther Sci. 2013;25(8):923-7.

27. Katsura Y, Yoshikawa T, Ueda S-Y, Usui T, Sotobayashi D, Nakao H, Sakamoto $\mathrm{H}$, Okumoto T, Fujimoto S. Effects of aquatic exercise training using waterresistance equipment in elderly. Eur J Appl Physiol. 2010;108(5):957-64.

28. Walia R. Comparison Between Land-based and Water-based Balance Training Exercise Program in Improvement of Balance in Community Dwelling Elderly Population. Indian J Physiother Occup Ther. 2012:6(3):221-27.

29. Cesar Barauce Bento P, Pereira G, Ugrinowitsch C, Rodacki ALF. The effects of a water-based exercise program on strength and functionality of older adults. J Aging Phys Act. 2012;20(4):469-70.

30. Bergamin M, Ermolao A, Tolomio S, Berton L, Sergi G, Zaccaria M. Waterversus land-based exercise in elderly subjects: effects on physical performance and body composition. Clin Interv Aging. 2013;8:1109.

31. Silva MR, Alberton CL, Portella EG, Nunes GN, Martin DG, Pinto SS. Waterbased aerobic and combined training in elderly women: effects on functional capacity and quality of life. Exp Gerontol. 2018;106:54-60.

32. Seyedjafari E, Sahebozamani M, Beyranvand R, Ebrahimipoor E, Razavi M. The effect of 8 weeks deep-aquatic exercises on static balance and lower body strength among elderly men. Int J Appl Exercise Physiol. 2017;6(1):86-98

33. Elbar O, Tzedek I, Vered E, Shvarth G, Friger M, Melzer I. A water-based training program that includes perturbation exercises improves speed of voluntary stepping in older adults: a randomized controlled cross-over trial. Arch Gerontol Geriatr. 2013;56(1):134-40.

34. Bento PCB, Lopes MFA, Cebolla EC, Wolf R, Rodacki ALF. Effects of waterbased training on static and dynamic balance of older women. Rejuvenation Res. 2015;18(4):326-31.

35. Alikhajeh $Y$, Hosseini SRA, Moghaddam A. Effects of hydrotherapy in static and dynamic balance among elderly men. Procedia Soc Behav Sci. 2012;46:2220-4.

36. Sanders ME, Takeshima N, Rogers ME, Colado JC, Borreani S. Impact of the SWEAT ${ }^{T M}$ water-exercise method on activities of daily living for older women. J Sports Sci Med. 2013:12(4):707.

37. Borg GA. Psychophysical bases of perceived exertion. Med Sci Sports Exerc. 1982;14(5):377-81.

38. Sherrington C, Tiedemann A, Fairhall N, Close JCT, Lord SR. Exercise to prevent falls in older adults: an updated meta-analysis and best practice recommendations. N S W Public Health Bull. 2011;22(4):78-83.

39. Pöyhönen $T$, Kyröläinen $H$, Keskinen $K L$, Hautala A, Savolainen J, Mälkiä E. Electromyographic and kinematic analysis of therapeutic knee exercises under water. Clin Biomech. 2001;16(6):496-504.

40. Lima CA, Ricci NA, Nogueira EC, Perracini MR. The berg balance scale as a clinical screening tool to predict fall risk in older adults: a systematic review. Physiotherapy. 2018;104(4):383-94.

41. Nightingale CJ, Mitchell SN, Butterfield SA. Validation of the timed up and go test for assessing balance variables in adults aged 65 and older. J Aging Phys Act. 2018;26(1):1-15.

42. Liu-Ambrose T, Khan KM, Eng JJ, Janssen PA, Lord SR, McKay HA. Resistance and agility training reduce fall risk in women aged 75 to 85 with low bone mass: a 6-month randomized, controlled trial. J Am Geriatr Soc. 2004;52(5):657-65.

43. Reed-Jones RJ, Dorgo S, Hitchings MK, Bader JO. Vision and agility training in community dwelling older adults: incorporating visual training into programs for fall prevention. Gait Posture. 2012;35(4):585-9.

44. Okubo Y, Schoene D, Lord SR. Step training improves reaction time, gait and balance and reduces falls in older people: a systematic review and meta-analysis. Br J Sports Med. 2017:51(7):586-93.

45. Arnold CM, Walker-Johnston J, Lanovaz JL, Lattimer LJ. Does fall arrest strategy training added to a fall prevention Programme improve balance, strength, and agility in older women? A Pilot Study. Physiotherapy Canada. 2017;69(4):323-32 
46. Holviala J, Häkkinen A, Alen M, Sallinen J, Kraemer W, Häkkinen K. Effects of prolonged and maintenance strength training on force production, walking, and balance in aging women and men. Scand J Med Sci Sports. 2014;24(1):224-33.

47. Chodzko-Zajko W, Schwingel A, Park CH. Successful aging: the role of physical activity. Am J Lifestyle Med. 2009;3(1):20-8.

48. Nilwik R, Snijders T, Leenders M, Groen BB, van Kranenburg J, Verdijk LB, van Loon $L$ J. The decline in skeletal muscle mass with aging is mainly attributed to a reduction in type II muscle fiber size. Exp Gerontol. 2013;48(5):492-8.

49. Kostka T. Quadriceps maximal power and optimal shortening velocity in 335 men aged 23-88 years. Eur J Appl Physiol. 2005;95(2-3):140-5.

50. Gafner SC, Bastiaenen CH, Ferrari S, Gold G, Terrier P, Hilfiker R. Allet L: hip muscle and hand-grip strength to differentiate between older fallers and non-fallers: a cross-sectional validity study. Clin Interv Aging. 2018;13:1.

51. Arvandi M, Strasser B, Volaklis K, Ladwig K-H, Grill E, Matteucci Gothe R, Horsch A, Laxy M, Siebert U, Peters A. Mediator effect of balance problems on association between grip strength and falls in older adults: results from the KORA-age study. Gerontol Geriatric Med. 2018;4:2333721418760122.

52. Blake AJ, Morgan K, Bendall MJ, Dallosso H, Ebrahim SBJ, Arie THD, Fentem $\mathrm{PH}$, Bassey EJ. Falls by elderly people at home: prevalence and associated factors. Age Ageing. 1988;17(6):365-72.

53. Musalek C, Kirchengast S. Grip strength as an Indicator of Health-related quality of life in old age-a pilot study. Int J Environ Res Public Health. 2017;14(12):1447.

54. Kerrigan DC, Lee LW, Collins JJ, Riley PO, Lipsitz LA. Reduced hip extension during walking: healthy elderly and fallers versus young adults. Arch Phys Med Rehabil. 2001;82(1):26-30.

55. Cristopoliski F, Sarraf TA, Dezan VH, Provensi CLG, Rodacki ALF. Transient effect of flexibility exercises in the hip joint on the gait of older women. Rev Bras Med Esporte. 2008;14(2):139-44.

56. Takeshima N, Rogers ME, Watanabe E, Brechue WF, Okada A, Yamada T, Islam MM, Hayano J. Water-based exercise improves health-related aspects of fitness in older women. Med Sci Sports Exerc. 2002;34(3):544-51.

57. Colado JC, Triplett NT, Tella V, Saucedo P, Abellán J. Effects of aquatic resistance training on health and fitness in postmenopausal women. Eur J Appl Physiol. 2009;106(1):113-22.

58. Pinto SS, Cadore EL, Alberton CL, Zaffari P, Bagatini NC, Baroni BM, Radaelli R, Lanferdini FJ, Colado JC, Pinto RS. Effects of intra-session exercise sequence during water-based concurrent training. Int J Sports Med. 2014; 35(01):41-8.

59. Bloomfield SA, Little KD, Nelson ME, Yingling VR. Position stand. Med Sci Sports Exerc. 2004;195(9131/04):3611-1985.

60. Gschwind YJ, Kressig RW, Lacroix A, Muehlbauer T, Pfenninger B, Granacher $U$. A best practice fall prevention exercise program to improve balance, strength / power, and psychosocial health in older adults: Study protocol for a randomized controlled trial. BMC Geriatr. 2013;13(1):105.

61. Baechle TR, Earle RW: Principios del entrenamiento de la fuerza y del acondicionamiento físico: Ed. Médica Panamericana; 2007.

62. Colado JC, Triplett NT. Monitoring the intensity of aquatic resistance exercises with devices that increase the drag force: an update. Strength Cond J. 2009;31(3):94-100.

63. Gianoudis J, Bailey CA, Ebeling PR, Nowson CA, Sanders KM, Hill K, Daly RM. Effects of a targeted multimodal exercise program incorporating high-speed power training on falls and fracture risk factors in older adults: a communitybased randomized controlled trial. J Bone Miner Res. 2014;29(1):182-91.

64. de Lacy-Vawdon CJ, Klein R, Schwarzman J, Nolan G, de Silva R, Menzies D, Smith BJ. Facilitators of attendance and adherence to group-based physical activity for older adults: a literature synthesis. J Aging Phys Act. 2018;26(1): 155-67.

65. Burke EJ. Individualized fitness program: using perceived exertion for the prescription of healthy adults. J Phys Educ Recreation. 1979;50(9):35-7.

66. Keteyian S, Ehrman J, Fedel F, Rhoads K. Heart rate-perceived exertion relationship during exercise in Orthotopic heart transplant patients. J Cardiopulm Rehabil Prev. 1990;10(8):287-93.

67. Melzer I, Elbar O, Tsedek I, Oddsson LIE. A water-based training program that include perturbation exercises to improve stepping responses in older adults: study protocol for a randomized controlled cross-over trial. BMC Geriatr. 2008;8(1):19.

68. Labelle H, Guibert R, Joncas J, Newman N, Fallaha M, Rivard CH. Lack of scientific evidence for the treatment of lateral epicondylitis of the elbow. An attempted meta-analysis. Bone Joint J. 1992;74(5):646-51.

Ready to submit your research? Choose BMC and benefit from:

- fast, convenient online submission

- thorough peer review by experienced researchers in your field

- rapid publication on acceptance

- support for research data, including large and complex data types

- gold Open Access which fosters wider collaboration and increased citations

- maximum visibility for your research: over $100 \mathrm{M}$ website views per year

At $\mathrm{BMC}$, research is always in progress.

Learn more biomedcentral.com/submissions 